\title{
REPRESENTATION OF ODOMETRY ERRORS ON OCCUPANCY GRIDS
}

\author{
Anderson A. S. Souza, Andre M. Santana, Ricardo S. Britto, Luiz M. G. Gonalves, Aderlardo A. D. Medeiros \\ Depatment of Computating Engineering and Automation, UFRN, Natal, Brazil \\ $\{$ abner, andremacedo, rbritto, lmarcos, adelardo $\} @ d c a . u f r n . b r$
}

Keywords: $\quad$ Mapping, Occupancy grid, Odometry.

Abstract: In this work we propose an enhanced model for mapping from sonar sensors and odometry that allows a robot to represent an environment map in a more suitable way to both the sonar sensory data and odometry system of the robot. We use a stochastic modelling of the errors that brings up reliable information. As a contribution, we obtain a final map that is more coherent with the reality of the original data provided by the robotic system. Practical experiments show the results obtained with the proposed modification to be trustable in such a way that this map can be used to provide previous knowledge to the mobile robot in order to perform its tasks in an easier and accurate way. Moreover, the map can help the robot to support unexpected situations inside of the environment

\section{INTRODUCTION}

In order for a robotic system to be efficient in the accomplishment of tasks, an important requirement is a correct spatial description of its underlying space that can be constructed from its own sensorial data. This description, a trustable map, makes possible a coherent interaction of the robot with its environment, so that it can perform its tasks efficiently and also can deal with unexpected situations like dynamical obstacles appropriately. The process of construction of this representation is generally named as mapping and the result is a map of environment.

Two main approaches are used to represent an environment in a map: the topological and the metric. In the topological approach, the environment map is represented by using graphs, in which the nodes are free spaces and the edges have information about connection among the nodes, for example, distance. Topological mapping makes easier the accomplishment of high level tasks, as navigation, with smaller computational cost.

In the metric approach, the geometry of the environment is defined in a more detailed way, presenting, accurately, the position of the objects inside of the environment, for instance walls, chairs and desks.
A common method used in the construction of metric maps is the occupancy grid. In this method, the environment is represented by a matrix in which each element represents a place in the environment that can be empty or occupied, or can be a unknown area.

In this work, we propose a mapping methodology with representation using an occupancy grid with a modification proposed in the model of the sonar sensor that embodies the uncertainty of the odometry system.

\section{RELATED WORKS}

There are many works in the literature dealing with occupancy grid. In 1987, Elfes (Elfes, 1987) proposed the occupancy grid method that is better formalized in his Ph.D thesis (Elfes, 1989). His work is implemented in two robots, Neptune and Terregator and is part of a more complete system, that integrates navigation and mapping based on sonars. This system is named Dolphin. Our current work also deals with sonars in the construction of the map in occupancy grid, but we have improved the sonar model by treating the noise of the system in a better way. 
Moravec (Moravec, 1988) proposes a system, in wich the occupancy grid map is based on sonar data and stereo data. Information from a sonar sensor array and from a stereo vision system is combined to build the metric map of environment.

In a subsequent work, Moravec (Moravec, 1996) introduces the idea of a map represented in a three dimensional occupancy grid. During the mapping, a sequence of stereo images is processed and the result of the mapping stored in a three dimensional array named evidence grid. The cells are initializes with zero value, indicating that there is no occupation evidence. After several sensor readings, the cells are filled out so that blocks of negative cells indicate free space, while positive blocks indicate obstacle. In his work, Moravec has used the theory of evidence of Dempster-Shafer. We use here the Bayesian approach also used by Elfes (Elfes, 1987).

Konolige (Konolige, 1997) has presented a method to treat the problems relative to the sonars in a more efficient way. Example of problems are the specular reflection and redundancy of reading. The proposed method is a mathematical refinement of the method introduced by Elfes (Elfes, 1987). The method is named as MURIEL (MUltiple Representation Independent Evidence Log).

In the Elfes' works, mapping is performed without considering dependence among the cell and its neighbours. This implicates in inconsistent maps when the mapping is performed in cluttered environments. In a more recent work, Thrun (Thrun, 2003) has introduced an advanced sensor model to deal with the problem of dependence among cells presented by the standard algorithm of Elfes (Elfes, 1987). The model introduced by Thrun (Thrun, 2003) verifies the values of occupation of the neighboring cells and then attributes the value of occupation to the current cell. Thrun has based his work on the Bayesian theory.

Thrun et. al. (Thrun et al., 2005) affirm that the main usefulness of the mapping with occupancy grid is in the post-processing, that is, in the map constructed. The map can be useful in several applications like, navigation, path planning, recognition of landmarks, obstacle avoidance and localization. Borenstein and Koren (Borenstein and Koren, 1991) for example, have implemented a method of obstacle avoidance in real time named VFF (Virtual Force Field). This method uses an occupancy grid map, obtained from sonar data, to define the localization of the obstacles inside of the environment.

In the Dutra's work (Dutra et al., 2003), a robot provided with an array of 24 sonars builds an occupancy grid map of its surroundings and store it in its internal memory. Later, this map is used for naviga- tion, but the results obtained by this work in both navigation and mapping were quite influenced by accumulated errors of the odometry system. In our work, we model the sonars taking into account the intrinsic errors also to the odometry system.

Other works in the literature exemplify the use of occupancy grid maps. Kong et. al. (Kong et al., 2006) has implemented a localization system based on EKF (Extended Kalman Filter) in which features inside the environment, as corners and flat surfaces, are detected. The information obtained about the features is integrated with an occupancy grid map known a priori to yield an accurate localization of the robot in the environment.

\section{THE STANDARD ALGORITHM OF OCCUPANCY GRID MAPPING}

The standard algorithm formalized by Elfes (Elfes, 1989), builds the map from both the sensorial data and the robot position (localization and orientation). The mathematical formulation of the occupancy grid mapping is derived from Equation (1), which gives the value of occupation of the whole map (Elfes, 1987; Thrun et al., 2005; Thrun, 2003).

$$
p\left(m \mid z_{1: t}\right)
$$

In this equation $m$ represents the obtained map, $z_{1: t}$ represents the set of measurements until the instant $t$. The continuos space of the environment is converted in a discrete space of cells, which form together an approximation of the environment. Thus, the map is defined as a finite set of cells $m_{x, y}$. Each cell posses a value, among 0 and 1, associated which corresponds to the state of the cell, occupied and empty, or can represent an unknown state. The value 0 means empty cell and 1 occupied cell. The notation $p\left(m_{x, y}\right)$ refers the probability of a cell of index $\langle x, y\rangle$ to be occupied.

The standard algorithm divides the problem of construction of the map in a set of smaller problems of estimate of the values of each cell $m_{x, y}$ separately.

$$
p\left(m_{x, y} \mid z_{1: t}\right)
$$

Due to reasons of numerical instabilities for probabilities near 0 or 1 , it is common to calculate the log-odds of $p\left(m_{x, y} \mid z_{1: t}\right)$ instead of $p\left(m_{x, y} \mid z_{1: t}\right)$. The log-odds is define for:

$$
l_{x, y}^{t}=\log \frac{p\left(m_{x, y} \mid z_{1: t}\right)}{1-p\left(m_{x, y} \mid z_{1: t}\right)}
$$


The probabilities are easily recovered from the logodds ratio:

$$
p\left(m_{x, y} \mid z_{1: t}\right)=1-\frac{1}{1+e^{l_{x, y}^{t}}}
$$

The value of the log-odds can be estimated recursively in any instant $t$ by the rule of Bayes applied on $p\left(m_{x, y} \mid z_{1: t}\right)$ :

$$
p\left(m_{x, y} \mid z_{1: t}\right)=\frac{p\left(z_{t} \mid z_{1: t-1}, m_{x, y}\right) p\left(m_{x, y} \mid z_{1: t-1}\right)}{p\left(z_{t} \mid z_{1: t-1}\right)}
$$

Supposing that we are mapping static environment, we can affirm that the current measurements of the sensors ones are independent of the last ones:

$$
p\left(z_{t} \mid z_{1: t-1}, m\right)=p\left(z_{t} \mid m\right)
$$

Because the map is decomposed in cells, this supposition is also extended all. We assume, then, the independence of each cell. Without take into account the occupation of the neighboring cells, we have:

$$
p\left(z_{t} \mid z_{1: t-1}, m_{x, y}\right)=p\left(z_{t} \mid m_{x, y}\right)
$$

This allows us to simplify the Equation (5).

$$
p\left(m_{x, y} \mid z_{1: t}\right)=\frac{p\left(z_{t} \mid m_{x, y}\right) p\left(m_{x, y} \mid z_{1: t-1}\right)}{p\left(z_{t} \mid z_{1: t-1}\right)}
$$

Applying the Total Probability Theorem to $p\left(z_{t} \mid m_{x, y}\right)$ we obtain:

$$
p\left(m_{x, y} \mid z_{1: t}\right)=\frac{p\left(z_{t} \mid m_{x, y}\right) p\left(m_{x, y} \mid z_{1: t-1}\right)}{\sum_{m_{x, y}} p\left(z_{t} \mid m_{x, y}\right) p\left(m_{x, y} \mid z_{1: t-1}\right)}
$$

The Equation (9) provides the probability of a cell $m_{x, y}$ to be occupied.

\section{THE PROPOSED MODEL OF THE SONAR}

In our work, we consider that noise is intrinsic to both data sources, sonar sensor and odometry system, in the construction of the map. The sonar sensors have internal and external features which arouse errors in their measurements. Usually, these errors are published by the manufacturers and made available to the public. In Figure 1, for example, we can verify some typical features of a sonar Polaroid series 6500, that are used in our work. This sonar presents higher sensibility in the area near to its main axis. Besides, it presents an error of absolute measurement of $+/-1 \%$.

Besides the typical errors of the sonar, we attempt to the errors accumulated by the odometry during the motion performed by the robot. The odometry calculates the robot's current pose in relation to the previous pose. With this, errors accumulate in an incremental way.

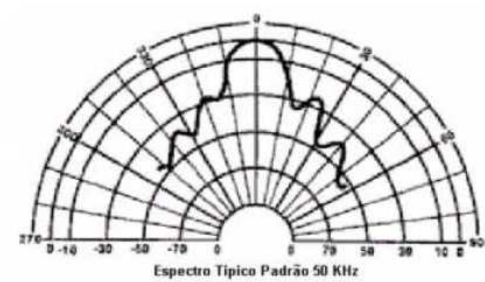

Figure 1: Features of the Polaroid sensor.

If we neglect these two sources of error, the final map will not be kept in accordance with the data originated from the robot, committing others applications like, navigation, path planning and others.

To deal whit those errors, we looked for a way of include them in the occupancy grid map representation. Thus, we modified the sonar sensor model of the standard algorithm, so that, in a probabilistic way, the typical errors of the sonars and odometry are incorporated to the value of occupation of the cell in the grid (Equation 10).

$p\left(z_{t} \mid m_{x, y}\right)=\frac{1}{2 \pi \sigma_{z_{t}} \sigma_{\theta_{t}}} \times e^{\left[-\frac{1}{2}\left(\frac{\left(D_{x, y}-z_{t}\right)^{2}}{\sigma_{z_{t}}^{2}}+\frac{\left(\theta_{x, y}-\theta\right)^{2}}{\sigma_{\theta_{t}}^{2}}\right)\right]}$

Where:

$z_{t}$ represents the sensor reading in the instant $t$; $\theta_{t}$ represents the orientation of the sensor; $\sigma_{z_{t}}$ represents the standard deviation regarding the error in the measured distance by the sensor;

$\sigma_{\theta_{t}}$ represents the standard deviation regarding the error in the orientation of the sensor;

$D_{x, y}$ represents the Euclidean distance between the sensor and the cell $m_{x, y}$;

$\theta_{x, y}$ represents the angle of orientation of the cell $m_{x, y}$.

The standard deviation regarding the error in the measured distance depends the value of the odometry error of a translational movement, that is,

$$
\sigma_{z_{t}}=z_{t} \times k+f
$$

Where:

$k$ is the factor of errors intrinsic of the sonar (in our case, $+/-1 \%$ );

$f$ is a function which describes the odometric systematic error, when the robot moves linearly.

The standard deviation regarding the error in the orientation angle of the sensor depends on the value of the odometry error of a rotational movement, that is,

$$
\sigma_{\theta_{t}}=\frac{\beta}{2}+g
$$

Where:

$\beta$ is the solid angle subtending the main lobe of the area of sensitivity; 
$g$ is a function which describes the odometric systematic error, when the robot moves in a rotational way.

Function $f$ in Equation 11 and function $g$ in Equation 12 were experimentally deduced from several data samples. Figure 2 shows a graph of the sensor model deduced.

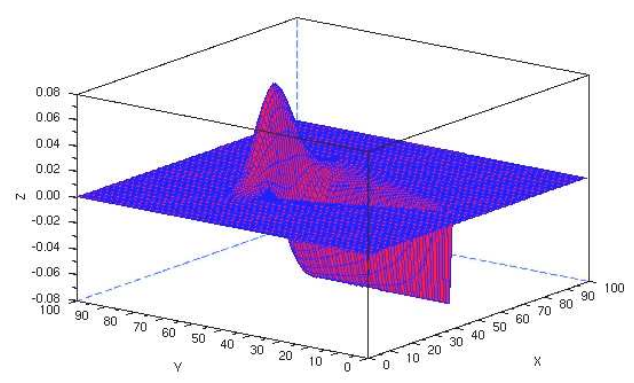

Figure 2: Graphics of the proposed model.

With this modification, the value of the probability of occupation of a cell passes to be weighed by the uncertainties in the real robot position, due to the odometry errors. Thus, the map is corrupted gradually, representing the real error.

\section{EXPERIMENTS}

To validate de proposed model, we have made some experiments with the mobile robot named Galatia, model Pioneer 3-AT of the ActivMedia Robotics, provided with two array sonar sensor (front and back) and odometry system (Figure 3).

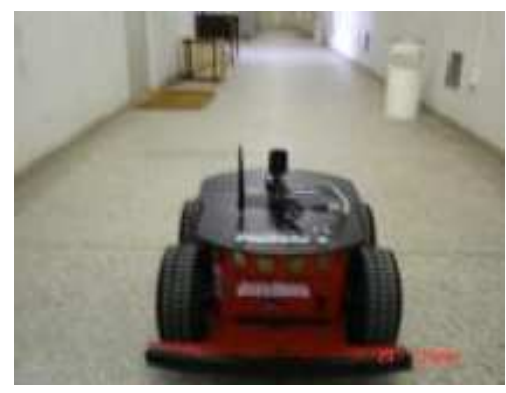

Figure 3: The robot Pioneer 3-AT.

The experiments are performed inside of the Department of Computing Engineering and Automation - UFRN, trying to map the corridors. The first experiment is performed using the standard algorithm of occupancy grid mapping without relying on the odometry errors. The result of this experiment can be seen in Figure 4.

Later, we perform the same experiment again using the probabilistic sonar model proposed here, to represent the systematic errors of the sonars and odometry (Figure 5).

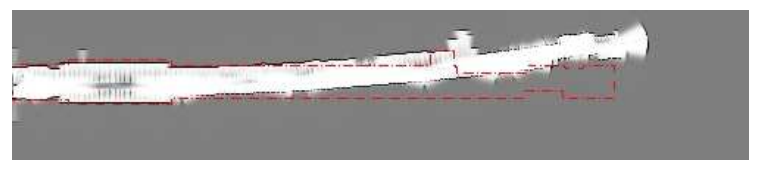

Figure 4: Map without representation of the errors.

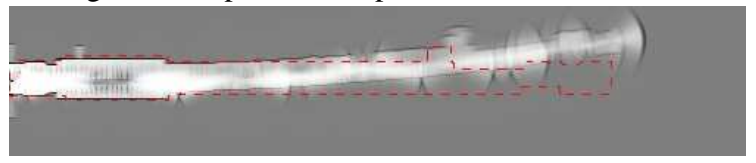

Figure 5: Corrupted map.

In the maps, the white cells represents free areas or no obstacles, the black cells represents occupied areas or obstacles and the grey cells represents the unknown areas. The sketched lines show the real contour of the corridors.

\section{CONCLUSIONS}

In this work, we propose a modification in the sensor model used in the standard algorithms based on occupancy grid mapping, including in the probabilistic model the intrinsic uncertainties of the robotic system. In order to consider these uncertainties in the mapping, we have found a way to include them in the representation of the map of the environment. Thus, we basically modify the sensor model so that, in a probabilistic way, the typical errors from sonars and odometry can be incorporated in the occupancy value of a cell. The model was tested in practice with the robot Pioneer 3-AT and demonstrated to be correct, giving the right expectation for the error

Based on the presented results, we can see that the proposed model supplies a more realistic way to represent a mapped environment using occupancy grid, being known that the originated information from the robot have errors, and what are really these errors. That is, the errors corrupt the quality of the map, showing in this way coherence whit the sensorial data.

As extension of this work, we will improve the treatment of the incoherent readings from the sonars, like speculate reflections and so. Furthermore, a way for relocation of the robot, when the errors grow a lot, will be studied and implemented.

\section{ACKNOWLEDGEMENTS}

We thanks CAPES, RNP and CNPq by the financial support. 


\section{REFERENCES}

Borenstein, J. and Koren, Y. (1991). The vector field histogram - fast obstacle avoidance for mobile robots. IEEE Journal of Robotics and Automation, 7(3):278288.

Dutra, P. R. C., de Souza, M. M., Andriolli, G. F., lvares, A. J., and Ferreira, J. C. E. (2003). Navmap: Um sistema para navegao por mapeamento do rob mvel nomad xr4000. In VI Simpsio Brasilieiro de Automao Inteligente (SBAI), Bauru, SP.

Elfes, A. (1987). Sonar-based real-world mapping and navigation. IEEE Journal of Robotics and Automation, 3(3):249-265.

Elfes, A. (1989). Occupancy Grid: A Probabilistic Framework for Robot Perception and Navigation. PhD thesis, Carnegie Mellon University, Pittsburg, Pennsylvania.

Kong, F., Chen, Y., Xie, J., Zhang, G., and Zhou, Z. (2006). Mobile robot localization based on extended kalman filter. In 6th World Congress on Inteligent Control and Automation, Dalian, China.

Konolige, K. (1997). Improved occupancy grids for map building. Autonomous Robots, (4):351-367.

Moore, R. and Lopes, J. (1999). Paper templates. In TEMPLATE'06, 1st International Conference on Template Production. INSTICC Press.

Moravec, H. P. (1988). Sensor fusion in certainty grids for mobile robots. AI Magazine, 9(2):61-74.

Moravec, H. P. (1996). Robot spatial perception by stereoscopic vision and $3 \mathrm{~d}$ evidence grid. Technical Report CMU-RI-TR-96-34, CMU Robotis Intitute, Pittsburgh, Pennsylvania.

Smith, J. (1998). The Book. The publishing company, London, 2nd edition.

Thrun, S. (2003). Learning occupancy grid maps with forward sensor models. Autonomous Robots, (15):111127.

Thrun, S., Burgard, W., and Fox, D. (2005). Probabilistic Robotics. MIT Press, Cambrige, Massachusetts, USA. 